\title{
A Privacy Preserving Repository for Securing Data across the Cloud
}

\author{
Ranjita Mishra \\ School of Computer Engineering, \\ KIIT University, \\ Bhubaneswar, India \\ ranjita_mishra86@yahoo.co.in
}

\author{
Sanjit Kumar Dash \\ Dept of IT, \\ College of Engineering and Technology, \\ Bhubaneswar, India \\ sanjitkumar303@gmail.com
}

\author{
Debi Prasad Mishra, Animesh Tripathy \\ School of Computer Engineering, \\ KIIT University, \\ Bhubaneswar, India \\ dpmishra.07@gmail.com, animesh_tripathy@kiit.ac.in
}

\begin{abstract}
Popularity of cloud computing is increasing day by day in distributed computing environment. There is a growing trend of using cloud environments for storage and data processing needs. Cloud computing is an Internet-based computing, whereby shared resources, software, and information are provided to computers and other devices on demand. However, adopting a cloud computing paradigm may have positive as well as negative effects on the data security of service consumers. This paper primarily highlights some major security issues existing in current cloud computing environments. The primary issue that has to be dealt with when talking about data security in a cloud is protection of the data. The idea is to construct a privacy preserving repository where data sharing services can update and control the access and limit the usage of their shared data, instead of submitting data to central authorities, and, hence, the repository will promote data sharing and privacy of data. This paper aims at simultaneously achieving data confidentiality while still keeping the harmonizing relations intact in the cloud. Our proposed scheme enables the data owner to delegate most of computation intensive tasks to cloud servers without disclosing data contents or user access privilege information.
\end{abstract}

Keywords- cloud, privacy, repository, integration, contextaware

\section{INTRODUCTION}

The impact during a major change in case of Information Technology industries is not always crystal clear and therefore a balanced wait and see attitude is perfect for an organization. However, some of the changes offer cost benefits, improvement of operations which in turn may offer significant strategic advantage. The benefits and the strategic advancement in these areas happen to be directly proportional to each other. The rise in these two factors will greatly impact the bottom line. The term cloud computing is known to many but a few have actually understood in depth of how it can be beneficial to them. For an organization, in order to jump to the cloud, it is of utmost importance to understand what, why, how and from whom. Since all cloud providers are not the same, many companies, for this reason are concerned over the protection of data as their entire data is being controlled by a central authority. In such a case companies may encrypt their data before even storing it in the cloud which in turn may lead some to offer greater data security and confidentiality than these companies. But, the fact to be considered is the level of security offered by various cloud providers which raises the security issue since all providers will not offer the same level of security. Generally speaking, security is usually improved by keeping the data in one centralized location.

\section{A. Cloud Overview}

Cloud computing is an Internet-based computing, whereby shared resources, software, and information are provided to computers and other devices on demand. It is an archetype shift following the shift from mainframe to clientserver. Details are extracted from the users, who no longer have need for expertise in, or control over, the technology infrastructure "in the cloud" that supports them. The term "cloud" is used as a metaphor for the Internet, based on the cloud drawing used in the past to represent the telephone network, and later to depict the Internet in computer network diagrams as an abstraction of the underlying infrastructure it represents. It is seen to have essential characteristics of selfservice, on-demand, location-independent and measured access to shared and elastic resources over the network. That is: ubiquitous, metered access to scalable network services.

Many formal definitions have been proposed in both academia and industry, the one provided by U.S. NIST (National Institute of Standards and Technology) [1] appears to include key common elements widely used in the Cloud Computing community:

Cloud computing is a model for enabling convenient, on demand network access to a shared pool of configurable computing resources (e.g., networks, servers, storage, applications, and services) that can be rapidly provisioned and released with minimal management effort or service provider interaction [2].

\section{B. Cloud Issues}

Cloud Computing is still in its infancy, current adoption is associated with numerous issues. In this section, we discuss some of the important issues associated with clouds.

Reliability: Stability of the data storage system is of important consideration in clouds. Generally, people worry about whether a cloud service provider is financially stable 
and whether their data storage system is trustworthy. Most cloud providers attempt to mollify this concern by using redundant storage techniques, but it is still possible that a service could crash or go out of business, leaving users with limited or no access to their data.

Data Backup: Cloud providers employ redundant servers and routine data backup processes, but some customers worry about being able to control their own backups. Many providers are now offering data dumps onto media or allowing users to back up their data through regular downloads.

Privacy: The Cloud model has been criticized by privacy advocates for the greater ease in which the companies hosting the Cloud services control and monitor communication and data stored between the user and the host company lawfully or unlawfully. There have been efforts to "harmonize" the legal environment by deploying local infrastructure and allowing customers to select "availability zones."

Security: Cloud service providers employ data storage and transmission encryption, user authentication, and authorization. Many clients worry about the vulnerability of remote data to criminals and hackers. Cloud providers are enormously sensitive to this issue and apply substantial resources to mitigate this problem.

Ownership: Once data has been relegated to the cloud, some worry about losing their rights or being unable to protect the rights of their customers. Many cloud providers address this issue with well-skilled user-sided agreements. According to the agreement, users would be wise to seek advice from their favorite legal representative.

Availability and Performance: Business organizations are worried about acceptable levels of availability and performance of applications hosted in the cloud.

Legal: There are certain points of concern for a cloud provider and a client receiving the service like location of the cloud provider, location of infrastructure, physical location of the data and outsourcing of the cloud provider's services etc.

The organization of our work is as follows. Section II focuses on data privacy security issues in the cloud and the advantage of protecting data. Section III briefly outlines the advantages of the privacy preserving repository pertaining to our construction. Section IV illustrates our thought towards approaching the construction. Section V presents our system architecture. In Section VI, we take a look at our proposed scheme in terms of its security and performance. We conclude this paper in Section VII.

\section{PRESERVING PRIVACY OF DATA IN THE CLOUD}

Security has been a serious issue of concern in the cloud. The main reason behind this is that both the customer data and the customer code lies in the provider premises. The following are some of the major issues related to security [3]:

- Server Access Security

- Database Access Security

- Internet Access Security

- Program Access Security

\section{- Data Privacy Security}

Of the above mentioned security issues we are focusing on the data privacy security issue. Privacy is the protection of transmitted data from passive attacks [4]. The objective is to ensure that the data required by customer is not being accessed by or not being disclosed to any unauthorized person in the cloud. The client has to assure themselves that the providers are executing their security responsibility. The general trend followed by customers for preserving privacy in cloud is as follows:

- Negotiate precise terms with their could provider

- Implement compensating controls where possible

- Assurance of the supplier regarding robustness of their service in preventing data from unauthorized access within a shared environment

- Establishment of an agreement particularly with regard to physical verification

- Get assurance from the cloud provider regarding security and compliance requirements

The responsibility for security is shared between the client and the service provider. To fight back against these issues we are proposing a repository for preserving privacy of data in cloud.

\section{OUR APPROACH TOWARDS PRESERVING PRIVACY OF DATA IN THE CLOUD}

In this paper, a privacy preserving repository is being presented for acceptance of integration requirements from clients to share data in the cloud and maintain their privacy, collect and integrate the appropriate data from data sharing services, and return the integration results to users. Our repository basically concentrates on the harmonizing operations and is beneficial in the following ways:

- The data sharing services in the cloud possess the ability to update and control the access and usages of their shared data. That is, data can be updated when required and it can be inferred who is using the data and in what way.

- The sharing of data in the cloud is done based on the need-to-share principle, which states that the dispatched information of the data is adequate to support clients' integration requirements, but carries no extra information of the data.

- The repository is limited to gathering data from data sharing services and combining the data to satisfy users' requirements. The repository will contain no other information apart from that required to deliver the results to the user and it cannot use this data for other purposes.

\section{AN INSPIRING EXAMPLE}

Let us consider a commercial information system which collaborates with multiple organizations through sharing and integrating data. The organizations may include company research institutes, project databases, a company database, 
and a location database for the purpose of studying the developments of popular projects being carried out at different locations. For the sake of simplicity, we assume that the system only communicates with one business research database T1 (PNAME; PNO) storing project names and corresponding project numbers, a project database T2 (ENO; PNO) storing the corresponding project numbers on which each employee works. It is assumed that a project can be worked upon by more than one employee, a company database T3 (ENO; PPLACE; PDEV) storing all employees' project progress, and a location database T4 (PNAME; PLOC) storing the location of where each project is being carried out. The databases' schemas and data are listed in Table 1. This example may be expressed in terms of four SQL queries shown in Table 2, where Q1, Q2, and Q3 generate three temporary tables, Tmp1, Tmp2, and Tmp3, respectively, and the last query, Q4, outputs the final results.

\section{TABLE1 DATABASES IN THE EXAMPLE}

Business Research
T1(PNAME, PNO)
\begin{tabular}{|c|c|}
\hline PNAME & PNO \\
\hline RD & P1 \\
\hline RD & P2 \\
\hline HR & P3 \\
\hline Purchasing & P4 \\
\hline
\end{tabular}

Company

T3(ENO,PPLACE,PDEV)

\begin{tabular}{|r|c|c|}
\hline ENO & PPLACE & PDEV \\
\hline ENO1 & P1 & Dev1 \\
\hline ENO2 & P1 & Dev2 \\
\hline ENO3 & P2 & Dev3 \\
\hline ENO4 & P3 & Dev4 \\
\hline
\end{tabular}

TABLE2 QUERIES REQUIRED BY THE EXAMPLE

\begin{tabular}{|l|l|}
\hline Q1 $\rightarrow$ Tmp1 & Q2 $\rightarrow$ Tmp2 \\
SELECT T1.PNO & SELECT T2.ENO \\
FROM T1 & FROM Tmp1, T2 \\
WHERE T1.PNAME="RD" & WHERE Tmp1.PNO="T2.PNO" \\
\hline Q3 $\rightarrow$ Tmp3 & Q4 \\
SELECT T4.PLOC & SELECT T3.PDEV \\
FROM T4 & FROM Tmp2, Tmp3, T3 \\
WHERE T4.PNAME="RD" & WHERE T3.ENO=Tmp2.ENO \\
& AND T3.PPLACE=Tmp3.PLOC \\
\hline
\end{tabular}

With the popular central warehouse solution [5], all data shown in Table 1 is collected by a central authority which can execute all queries. However, our repository is allowed to collect only the needed information. On the other hand our repository will randomize Q1's result and make the randomized result still usable for Q2 because the repository needs some extra information to execute queries, such as Q1's result, which is needed by Q2 as an input, Although existing privacy preserving query processing approaches, such as [6], [7], [8], [9], [10], [11], [12], can evaluate a query on randomized data, none of them can handle a series of queries, where some queries need other queries' results as inputs, such as Q2 in this motivating example. In [13], [14], a symmetric searchable encryption scheme and an asymmetric searchable encryption scheme are proposed to store users' data in a third party. Here, in our paper to protect $\mathrm{Q} 1$ 's result i.e. $\{\mathrm{P} 1 ; \mathrm{P} 2\}$ without disabling $\mathrm{Q} 2,\{\mathrm{P} 1 ; \mathrm{P} 2\}$ is replaced by $\{\mathrm{H}(\mathrm{P} 1) ; \mathrm{H}(\mathrm{P} 2)\}$, where $\mathrm{H}$ is a hash function. Because the hashed patterns will usually remain unique, the repository can evaluate Q2 by comparing $\mathrm{H}(\mathrm{Tmp} 1$;PNO) and $\mathrm{H}(\mathrm{T} 2 ; \mathrm{PNO})$. This simple hash solution can avoid the need for our repository to know Q1's results, but still keep the mapping relation between non RD projects and employees' ENOs. Since H(P3) does not appear in the Q1's hashed result $\{\mathrm{H}(\mathrm{P} 1) ; \mathrm{H}(\mathrm{P} 2)\}$, our repository can find that the employee with ENO4 is not working on $\mathrm{RD}$. To protect the privacy of such information, the concept of Context-Aware Data Sharing [15] is used to randomize Q1's result. The contextawareness implies that when a business research institute shares its database T1 with our repository, it should know that its project number (PNO) data will be used to match the project number data from T2. While the simple hash solution only randomizes the items in Q1's result (i.e., P1; P2), our Context-Aware Data Sharing concept randomizes all PNOs in T1, but ensures that only $\mathrm{P} 1$ and $\mathrm{P} 2$ can be used to evaluate Q2. Hence, the mapping between non RD projects and ENOs are well protected.

In this paper, we will be focusing on the above example to illustrate how our repository is being used for studying the developments of various projects at different locations and how the data cannot disclose any additional information about the data of databases T1, T2, T3, and T4.

\section{PRELIMINARIES}

\section{A. System Architecture and Assumptions}

In continuing data integration systems, the concept of a central and trusted authority collecting all data from data sharing services and computing the integration results for users based on the data collected is usually not valid for data sharing services across various organizations. In our system, as in the Fig. 1 given below, our repository will collect only that data required for generating users' requests. It is assumed that our repository will correctly construct the query plans for users' integration requirements, decompose the query plans, discover and fetch data from distributed data sharing services, assimilate all data together, and, finally, return the final results to users. Further, we assume that our repository is granted the access to the shared data by all data sharing services, and all shared data is well protected. Because the data sharing services use our context-aware data sharing concept, our repository cannot learn any extra information from the inferential relations of the information it obtains during the process. Our repository consists of two components: the query plan wrapper and the query plan executor. The query plan wrapper is responsible for scrutinizing integration requirements and constructing query plans for the query plan executor. Since the wrapper development and optimization have been extensively studied [16], [17], [18], [19], [20], [21], we assume that the query 
plan wrapper can select data sharing services and construct a query plan graph[15] from users' integration requirements. The decomposition of the query plan graph into a set of small sub- graphs for each data sharing service [15] is used to guide data sharing services to prepare shared data. The query plan executor is responsible for executing query plans to fetch data from data sharing services and producing the final results. In this paper, we are proposing a secure query plan executor which can execute query plans without additional information about the data of data sharing services.

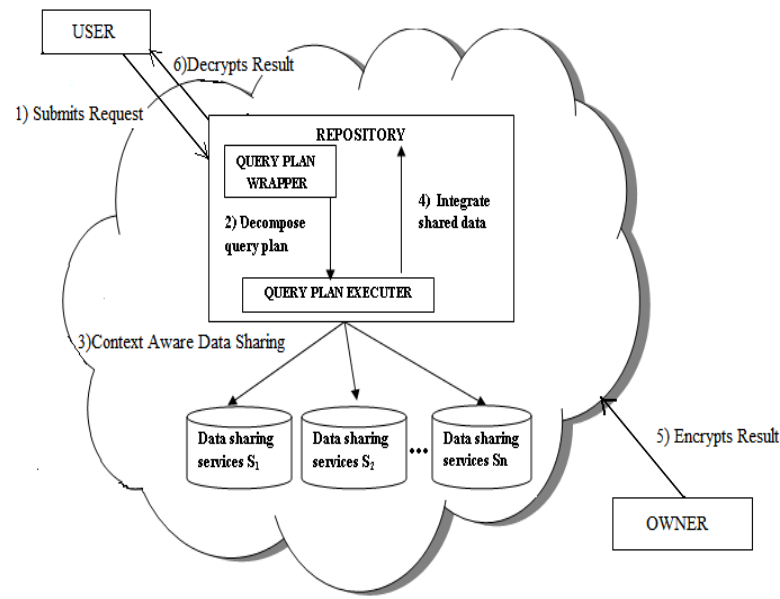

Fig. 1 Our Privacy Preserving Repository for Securing Data across the Cloud

\section{B. Privacy Preserving Query Plan with Repository}

To formulate the privacy preserving data integration across data sharing services in the cloud, it is needed to define the query plan [15]:

Definition of Query Plan: A query plan $\mathrm{P}$ is a partially ordered set of queries $\left(\mathrm{p}_{1} ; \mathrm{p}_{2} ;{ }_{\ldots} \ldots \ldots ; \mathrm{p}_{\mathrm{m}}\right)$ with two properties:

1) Each $p_{i}$ can be evaluated only after all of its precedent queries have been evaluated

2) The data from the data sharing services can be directly used by each $p_{i}$ or the precedent queries' outputs can be used as inputs

The output of $p_{i}$ with no succeeding queries is the final result of $\mathrm{P}$, and all other queries' outputs are intermediate results.

The above definition indicates that a query plan $\mathrm{P}$ has a much richer structure than a single query or a set of independent queries. First, there is a partial order relation among queries in $\mathrm{P}$. Second, only the outputs of queries in $\mathrm{P}$ without successive queries constitute the final result and all other intermediate results should be protected. Consequently, we have the following definition:

Definition of Privacy Preserving Repository: For a query plan $\mathrm{P}=\left(\mathrm{p}_{1} ; \mathrm{p}_{2} ; \ldots \ldots ; \mathrm{p}_{\mathrm{m}}\right)$ and a repository $\mathrm{REP}$, where REP is a privacy preserving repository for data integration if REP executes $\mathrm{P}$ in a privacy preserving manner as follows:
1) REP has only P's final result encrypted with user's public key and has no information on P's intermediate results

2) REP cannot use the data shared for P to evaluate any other queries.

\section{OVERVIEW OF OUR APPROACH}

Our goal is to build up a repository to facilitate the data integration and sharing across cloud along with preservation of data confidentiality. In this section, we will present the process of the data integration via our privacy preserving repository REP. The process can be summarized as follows: Step 1: The user sends his/her public key $P_{k}$ and the requirements about data integration to our repository REP. Step 2: The query plan wrapper of REP analyzes the user's integration requirements and converts them to a query plan graph $\mathrm{G}$, and then decomposes $\mathrm{G}$ to a set of sub-graphs $\left(\mathrm{G}_{1} ; \mathrm{G}_{2} ; \ldots \ldots \mathrm{G}_{\mathrm{m}}\right)$ and sends the sub-graphs to the query plan executor. Every sub graph $\mathrm{G}_{\mathrm{i}}$ represents the context of one data sharing service for conducting context-aware data sharing.

Step 3: For every $G_{i}$, the query plan executor looks for the corresponding data sharing service $S_{i}$ and sends $G_{i}$ to $S_{i}$, which prepares the data using the Context-Aware Data Sharing concept and returns all randomized data to the query plan executor.

Step 4: The query plan executor integrates all returned data to execute the $G$ and outputs the results FinalRes of user's request, which is encrypted with the user's public key $\mathrm{P}_{\mathrm{k}}$.

Step 5: REP sends Final Result to the user who then decrypts it with his/her secret key $\mathrm{S}_{\mathrm{k}}$.

\section{CONCLUSION}

In an emerging discipline, like cloud computing, security needs to be analyzed more frequently. With advancement in cloud technologies and increasing number of cloud users, data security dimensions will continuously increase. In our paper we are laying stress on the security issue in the cloud. The proposed scheme is probably secure under the standard security model [22]. In addition, our proposed scheme is able to support user accountability with minor extension. Whether you are assembling, managing or developing on a cloud computing platform, you need a cloud-compatible database. The shared-disk database architecture [23], on the other hand, does support elastic scalability. It also supports other cloud objectives such as lower costs for hardware, maintenance, tuning and support. It delivers high-availability in support of Service Level Agreements (SLAs). As with every tectonic shift in technology, there is a Darwinian ripple effect as we realize which technologies support these changes and which are relegated to legacy systems. Because of their compatibility, cloud computing will usher in an ascendance of the shared-disk database. The privacy preserving repository delivers the critical capabilities required for a robust, cost-effective, and secure cloud security implementation. 


\section{FUTURE WORK}

In future we will be coming up with a secure development scheme of the hash function and its implementation in public key cryptography for maintaining the privacy of data in the cloud.

\section{REFERENCES}

[1] P. Mell and T. Grance, "Draft-NIST working definition of cloud computing - v15," 21. Aug 2005, 2009.

[2] M. Armbrust, A. Fox, R. Griffith, A. Joseph, R. Katz, A. Konwinski, G. Lee, D. Patterson, A. Rabkin, and I. Stoica, "Above the clouds: A Berkeley view of cloud computing," EECS Department, University of California, Berkeley, Tech. Rep. UCB/EECS-2009-28, 2009.

[3] Rakshit, A. , et. Al, "Cloud Security Issues", 2009, IEEE International Conference on Services Computing

[4] Stallings, W., "Cryptography and Network Security", $3^{\text {rd }}$ Edition, Pearson Education, New Delhi, 2003, p8.

[5] Kamber, et al., "Data Mining Concepts and Techniques", $2^{\text {nd }}$ Edition, Elsevier, New Delhi, 2009, p12-13

[6] R. Agrawal, A.V. Evfimievski, and R. Srikant, "Information Sharing across Private Databases," Proc. ACM SIGMOD Int'l Conf. Management of Data (SIGMOD '03), pp. 86-97, 2003.

[7] R. Agrawal, J. Kiernan, R. Srikant, and Y. Xu, "Order-Preserving Encryption for Numeric Data," Proc. ACM Int'l Conf. Management of Data (SIGMOD '04), pp. 563-574, 2004.

[8] M. Bellare, A. Boldyreva, and A. O’Neill,'Deterministic and Efficiently Searchable Encryption," Advances in Cryptology (CRYPTO '07), pp. 535-552, 2007.

[9] T. Ge and S.B. Zdonik, "Answering Aggregation Queries in a Secure System Model," Proc. 33rd Int'l Conf. Very Large Data Bases (VLDB '07), pp. 519-530, 2007.

[10] Y. Lindell and B. Pinkas, "Privacy Preserving Data Mining," J. Cryptology, vol. 15, no. 3, pp. 177-206, 2002.

[11] M. Scannapieco, I. Figotin, E. Bertino, and A.K Elmagarmid, "Privacy Preserving Schema and Data Matching," Proc. ACM Int'l Conf. Management of Data (SIGMOD '07), pp. 653-664, 2007.

[12] L. Xiong, S. Chitti, and L. Liu,'Preserving Data Privacy for Outsourcing Data Aggregation Services," ACM Trans. Internet Technology, vol. 7, no. 3, pp. 17-45, 2007.

[13] D. Boneh, G.D. Crescenzo, R. Ostrovsky, and G. Persiano, "Public Key Encryption with Keyword Search," Advances in Cryptology (EUROCRYPT '04), pp. 506-522, 2004.

[14] D.X. Song, D. Wagner, and A. Perrig,"Practical Techniques for Searches on Encrypted Data," Proc. IEEE Symp. Security and Privacy (S\&P '00), pp. 44-55, 2000. Yau, Stephan S., et. al, “A Privacy Preserving Repository for Data Integration across Data Sharing Services", IEEE Transactions on Services Computing, Vol 1, No-3, July-September 2008

[15] Yau, Stephan S., et. al, "A Privacy Preserving Repository for Data Integration across Data Sharing Services", IEEE Transactions on Services Computing, Vol 1, No-3, July-September 2008

[16] S. Adali, K.S. Candan, Y. Papakonstantinou, and V.S Subrahmanian, "Query Caching and Optimization in Distributed Mediator Systems," Proc. ACM Int'l Conf. Management of Data (SIGMOD '96), pp. 137-148, 1996.
[17] Y. Arens, C.A. Knoblock, and W. Shen,"'Query Reformulation for Dynamic Information Integration," J. Intelligent Information Systems, vol. 6, no. 2, pp. 99-130, 1996.

[18] Z.G. Ives, D. Florescu, M. Friedman, A.Y. Levy, and D.S. Weld,"An Adaptive Query Execution System for Data Integration," Proc. ACM Int'l Conf. Management of Data (SIGMOD '99), pp. 299- 310, 1999

[19] K.P. Fischer, U. Bleimann, W. Fuhrmann, and S.M. Furnell, "Security Policy Enforcement in BPEL-Defined CollaborativeBusiness Processes," Proc. 23rd Int'l Conf. Data Eng. Workshop, pp. 685-694, 2007.

[20] A.Y. Levy, A. Rajaraman, and J.J. Ordille, "Querying Heterogeneous Information Sources Using Source Descriptions," Proc. 22th Int'l Conf. Very Large Data Bases (VLDB '96), pp. 251262, 1996.

[21] B. Yu, G. Li, K.R. Sollins, and A.K.H. Tung,"'Effective KeywordBased Selection of Relational Databases," Proc. ACM Int'l Conf. Management of Data (SIGMOD '07), pp. 139-150, 2007.

[22] Shucheng Yu., Cong Wangt, Kui Ren , and Wenjing Lou , "Achieving Secure, Scalable, and Fine-grained Data Access Control in Cloud Computing", IEEE INFOCOM 2010 Proceedings

[23] Hogan, M., "Cloud Computing and Databases", Scale DB Inc., 2008

Ranjita Mishra received the B.Tech. Degree in Information Technology from Biju Patnaik University of Technology, Odisha, India, in 2009 and pursuing M.Tech. Degree in Computer Science and Engineering with specialization in Information Security at Kalinga Institute of Industrial Technology, Bhubaneswar, India. Her research interests include Cloud Computing, Data Mining and Cryptography.

Sanjit Kumar Dash received the B.Tech. Degree in Information Technology from Biju Patnaik University of Technology, Odisha, India, in 2004 and pursuing M.Tech. Degree in Computer Science and Engineering at Institute of Technical Education and Research, Bhubaneswar, India. He is also working as a faculty member at the Information Technology Department, College of Engineering and Technology, Bhubaneswar, India. His research interests include Cloud Computing, Sensor Network, and Mobile Computing.

Debi Prasad Mishra received the B.Tech. Degree in Information Technology from Biju Patnaik University of Technology, Odisha, India, in 2007 and pursuing M.Tech. Degree in Computer Science and Engineering with specialization in Information Security at Kalinga Institute of Industrial Technology, Bhubaneswar, India. His research interests include Network Security and Web Technologies.

Animesh Tripathy is currently pursuing his Research in Intelligent Database Systems. He has completed his Bachelor of Engineering in Computer Engineering and Master of Technology in Computer Science \& Engineering from University of Calcutta. Recently he has submitted his $\mathrm{PhD}$ in Intelligent Mining of Database Systems to Utkal University. Presently he is working as a Professor in Computer Science Department, KIIT University, Bhubaneswar, India. He has published many innovative research papers in International Journals \& Conferences. His major strength lies in Spatial Database, Image Analysis \& Data Mining. 